\title{
Clima organizacional y desempeño laboral docente en instituciones educativas de nivel primario y secundario
}

\section{Organizational climate and teaching job performance in elementary and high school educational institutions}

\author{
Roberto Paco Ccora' \\ Universidad Continental \\ roberto.pacoc@urp.pe
}

\author{
José María Matas Castañeda² \\ Universidad Continental \\ imatas@continental.edu.pe
}

\section{RESUMEN}

El objetivo fue determinar la existencia de una relación significativa entre el clima organizacional y el desempeño laboral docente en la Institución Educativa Particular San PíoX X la Institución Educativa Estatal Rosa de Lima de San Jerónimo de la provincia de Huancayo. La investigación fue de tipo básico descriptivo correlacional. El tipo de muestra fue no probabilístico e incluyó 110 docentes entre varones y mujeres del nivel primario y secundario; 60 docentes de la I.E.P San Pío X y 50 de la I.E.E. Rosa de Lima de San Jerónimo. Los instrumentos aplicados fueron las escalas de clima social en el trabajo (WES), que evalúa el ambiente social existente en diversos tipos y centros de trabajo, y el inventario de desempeño laboral. Los resultados de la investigación determinaron la existencia de una relación positiva y significativa, entre el clima organizacional y el desempeño laboral docente en la I.E.P. San Pio X y la I.E.E. Rosa de Lima, con un valor $r$ de Pearson $=0,71$. En conclusión, el clima organizacional es un factor determinante en el desempeño laboral docente e implica una referencia de los miembros respecto a su estar en la organización. Por tanto, un clima favorable conllevará a la predisposición de un trabajo óptimo, lo cual tendrá un impacto positivo en la enseñanza, aprendizaje y la construcción de nuevos conocimientos.

Palabras clave: Clima organizacional, aprendizaje, clima social, Institución Educativa.

\section{ABSTRACT}

The aim was to determine if there was a significant relationship between organizational climate and job performance in Private School San Pío $X$ teachers and Public Educational Institution Rosa de Lima in San Jeronimo, Huancayo province. The research was basic, descriptive and correlational. The sampling type was not probabilistic and included 110 teachers in total between male and female from elementary and high school levels; 60 teachers from San Pio X Private School and 50 teachers from Rosa de Lima Public Educational Institution in San Jeronimo. The instruments applied were: Social climate scales at work (WES), which assess the existing social environment in various workplaces and the job performance inventory. The research findings determined a significant relationship between organizational climate and job performance of teachers from San Pio X Private School Institution and Rosa de Lima Public Educational Institution, from San Jeronimo ( $r$ Pearson value of 0,71 ). The organizational climate is a determining factor in teacher's competencies and factors affecting their performance; and involves a constant reference to their members regarding their staying in the organization. Therefore, a favorable climate within the scope of work will lead to the predisposition of a great job, which has a positive impact on teaching, student learning and the generation of new knowledge.

Keywords: Organizational climate, teacher job performance, social climate, particular educational institution, state educational institution.

Historial del artículo:

Recibido: 8 de abril de 2015. Aprobado: 3 de octubre de 2015. Disponible en línea: 30 de diciembre de 2015

1 Bachiller de la Escuela de Psicología de la Universidad Continental.

2 Licenciado en Ciencias Empresariales de la Universidad Ramon Llull, Barcelona, España. Docente en la Universidad Continental. 


\section{INTRODUCCIÓN}

Desde hace décadas se estudia la forma de mejorar y optimizar el desempeño laboral y se investiga los factores que influyen en este, el clima organizacional es un conjunto de características permanentes que describen una organización, la distinguen de otra e influye en el comportamiento de las personas que la forman (1), tiene un componente multidimensional de elementos que pueden descomponerse en términos de estructuras organizacionales, tamaño de la organización, modos de comunicación, estilos de liderazgo de la dirección, entre otros; también se explica que este comprende un espacio más amplio y flexible de la influencia ambiental sobre la motivación. El clima organizacional es la cualidad o propiedad del ambiente organizacional que perciben o experimentan los miembros de una organización y que influyen en su comportamiento (2).

La carencia de un buen clima organizacional afecta no solo el rendimiento del trabajador sino también la salud y el bienestar personal y familiar. Sin embargo, como en toda organización se presentan, problemas en el contexto laboral, generalmente relacionados con la dimensión humana, tales como problemas a nivel de relaciones interpersonales, falta de identidad y compromiso con el centro laboral, estrés, insatisfacción laboral, entre otros. El clima organizacional es un tema de gran importancia hoy en día para casi todas las organizaciones, las cuales buscan un continuo mejoramiento del ambiente de su organización para así alcanzar un mejoramiento de la productividad, sin perder de vista el recurso humano; estudios recomiendan en toda empresa establecer programas de higiene laboral tomando en cuenta el ambiente físico de trabajo, la aplicación de la ergonomía, salud ocupacional y trabajo en equipo en el que se incluya la cooperación, la asistencia, disciplina y conflicto (3).

El clima organizacional es de mucha relevancia para los docentes de instituciones públicas y privadas ya que en esta se puede observar conflictos laborales de diversa índole, permitiendo así el clima laboral desfavorable en el trabajo que se imparte día a día.

La investigación responde a la pregunta: ¿¿Qué grado de relación existe entre el clima organizacional y el desempeño laboral de los docentes de la I.E.P. San Pío $X$ y la I.E.E. Rosa de Lima de San Jerónimo?. El objetivo de la investigación consistió en determinar el grado de relación existente entre el clima organizacional y el desempeño laboral docente de la I.E.P. San Pío X y la I.E.E. Rosa de Lima de San Jerónimo. La hipótesis planteada fue que, sí existe relación significativa entre el clima organizacional y el desempeño laboral en los docentes de ambas instituciones educativas.

Actualmente, debido a los procesos de cambios y las nuevas tecnologías que surgen en el mercado, se ha propiciado el desarrollo de más habilidades, destrezas y conocimientos. Las organizaciones se han visto en la necesidad de implementar cambios en su estrategia laboral a la hora de enfrentar los retos que se les presentan.

En este contexto, la productividad y el manejo del capital humano en las organizaciones se convierten en elementos clave de sobrevivencia; por tanto, la coordinación, dirección, motivación y satisfacción del personal son aspectos cada vez más importantes del proceso administrativo. Entre ellos, la satisfacción del trabajador ocupa un lugar preferente, ya que la percepción positiva o negativa que los trabajadores mantienen con respecto a su trabajo influye en la rotación de personal, ausentismo, aparición de conflictos y en otras áreas esenciales de la organización.

Todas las organizaciones tienen propósito, estructura y una colectividad de personas, y están conformadas por un grupo de elementos interrelacionados entre sí, tales como estructura organizacional, procesos que se dan dentro de ellas, y conductas de los grupos e individuos.

\section{MATERIAL Y MÉTODOS}

La investigación fue de carácter básico descriptivo de nivel correlacional. Con el fin de demostrar la relación significativa entre el clima institucional y el desempeño laboral de los docentes de dichas instituciones. Para ello se empleó el test de escala del clima social en el trabajo (WES) formado por diez sub escalas que evalúan tres dimensiones fundamentales: relaciones, autorrealización y la estabilidad/cambio, lo cual nos permitió tener el diagnóstico del clima institucional de los docentes de la muestra (4). Asimismo, para evaluar el desempeño de los docentes en sus instituciones, se usó el inventario de desempeño laboral de cuatro dimensiones con indicadores para medir el compromiso, motivación, satisfacción laboral y eficiencia.

La población de estudio estuvo constituida por todos los docentes de los dos centros educativos. La muestra fue conformada por 110 docentes de ambos géneros nivel primario y secundario, 60 de la I.E.P. San Pío $X$ y 50 de la I.E.P. Rosa de Lima de San Jerónimo, en ambos casos integrados por varones y mujeres.

En la selección definitiva de los elementos se tuvieron en cuenta los siguientes criterios psicométricos: 1) La elección de alternativas (verdadero - falso) debería ser tan próxima a $50 \%-50 \%$ como fuese posible para evitar elementos característicos de ambientes de trabajo poco comunes; 2) los elementos deberían presentar correlaciones más altas con su propia subescala 
que son cualquier otra; 3) cada escala debería tener igual número de elementos que puntuasen en las dos alternativas (verdadero-falso) para controlar los sesgos de moderadas; 4) las subescalas deberían presentar intercorrelaciones bajas o moderadas; y 5) cada elemento y cada subescala deberían ser discriminativos en distintos ambientes de trabajo.
Tabla $N^{\circ}$ 1: Clima social docente de las Instituciones Educativas Rosa de Lima y San Pío X.

\begin{tabular}{lcc}
\hline \multicolumn{1}{c}{ Clima social } & Frecuencia & $\%$ \\
\hline I. E. E. Rosa de Lima & 1312 & 48,6 \\
I. E. P San Pío X & 1387 & 51,4 \\
Total & 2699 & 100,0 \\
\hline
\end{tabular}

Para la recolección de datos se utilizaron las técnicas psicométricas, que consiste en la aplicación de test o

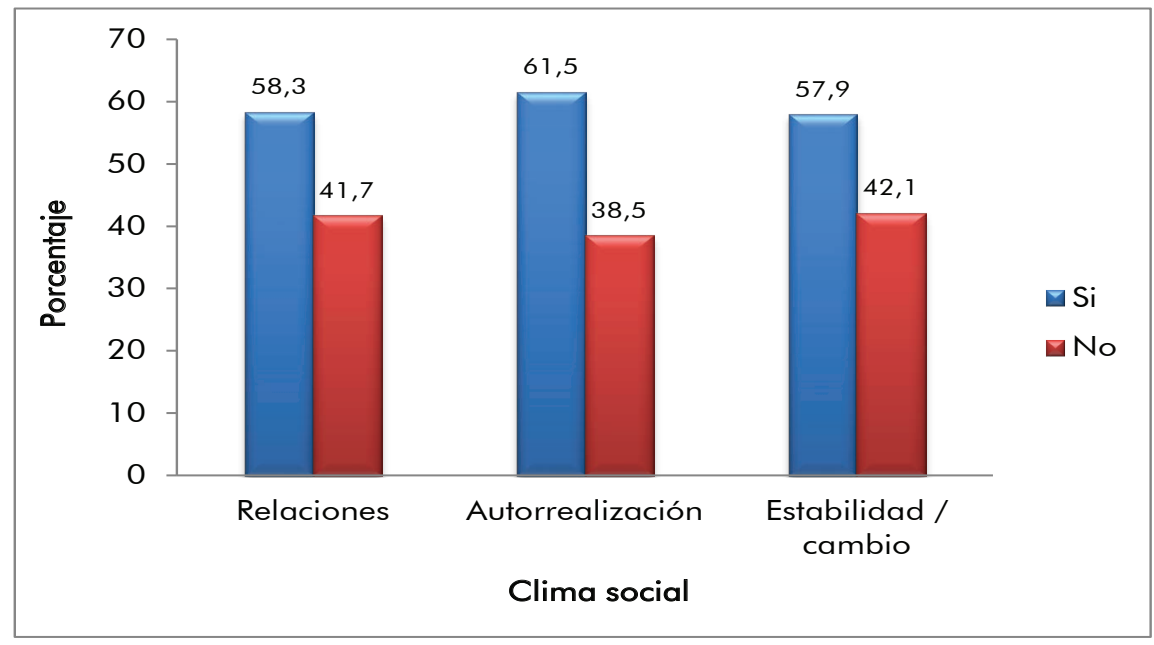

Figura $N^{\circ}$ 1: Clima social docente por conceptos en la I.E.E. Santa Rosa de Lima.

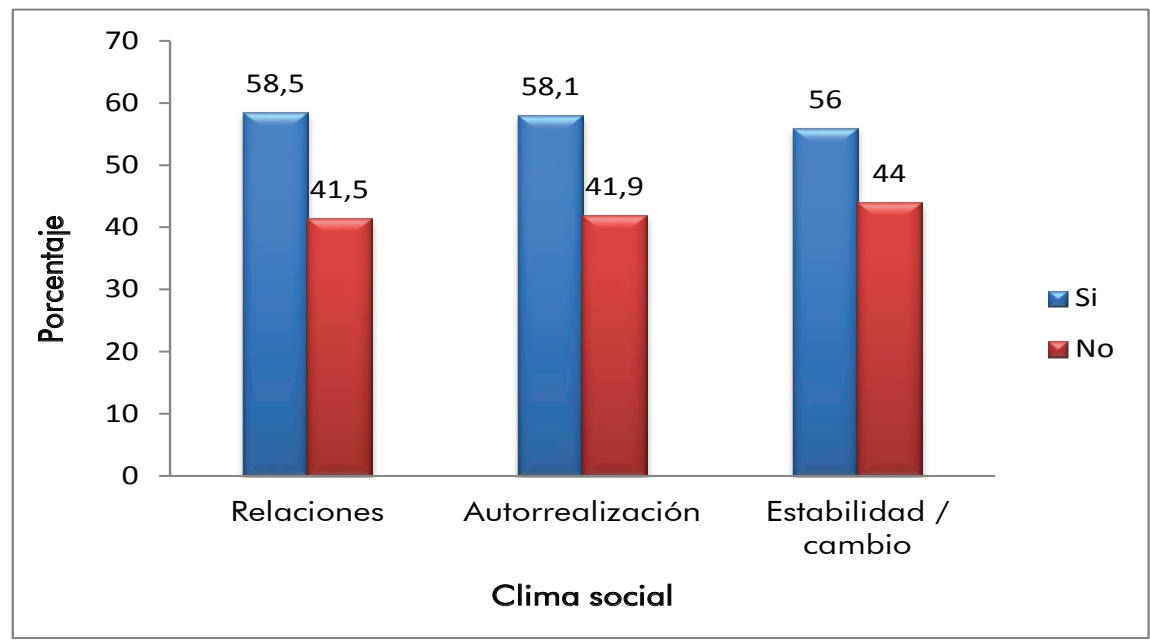

Figura $\mathrm{N}^{\circ}$ 2: Clima social docente por conceptos en la I.E.P. San Pío X.

pruebas psicológicas, las cuales nos permitió obtener datos con rapidez las dimensiones acerca de las personalidades de los sujetos de la muestra de estudio, sobre el clima institucional y el desempeño laboral.

\section{RESULTADOS}

Se determinó que existe una relación positiva y significativa entre el clima organizacional y el desempeño laboral docente en las I. E. P. San Pio X y la I. E. E. Rosa de Lima de San Jerónimo, con un valor de $r$ de Pearson $=0,71$ y un IC (Intervalo de Confianza) de $95 \%$, dicha relación significativa se dio diferenciando tanto el sexo como la Institución Educativa.

Si bien hay una relación muy estrecha y significativa entre clima social y desempeño docente en las dos instituciones educativas. Los datos de la tabla 1 indican 
que el mayor clima social docente se manifiesta en la I.E.P. San PíoX (51,4 \% sobre 48,6\%). En la figura 1 y 2 se muestra que, para la evaluación del clima social en cada una de las instituciones educativas, se aplicaron preguntas agrupadas en tres conceptos: relaciones, autorrealización y estabilidad/cambio. Al comparar las dos Instituciones Educativas, se observan resultados similares para el concepto de Relaciones. Sin embargo, las diferencias están en la Autorrealización y en la Estabilidad/cambio, ya que en la I.E.E. Santa Rosa de
Tabla № 2: Desempeño docente de las Instituciones Educativas Rosa de Lima y San Pío X.

\begin{tabular}{lcr}
\hline Desempeño docente & $\mathrm{Fi}$ & $\%$ \\
\hline I. E. E. Rosa de Lima & 3332 & 51 \\
I. E. P San Pío X & 3148 & 49 \\
Total & 6480 & 100,0 \\
\hline
\end{tabular}

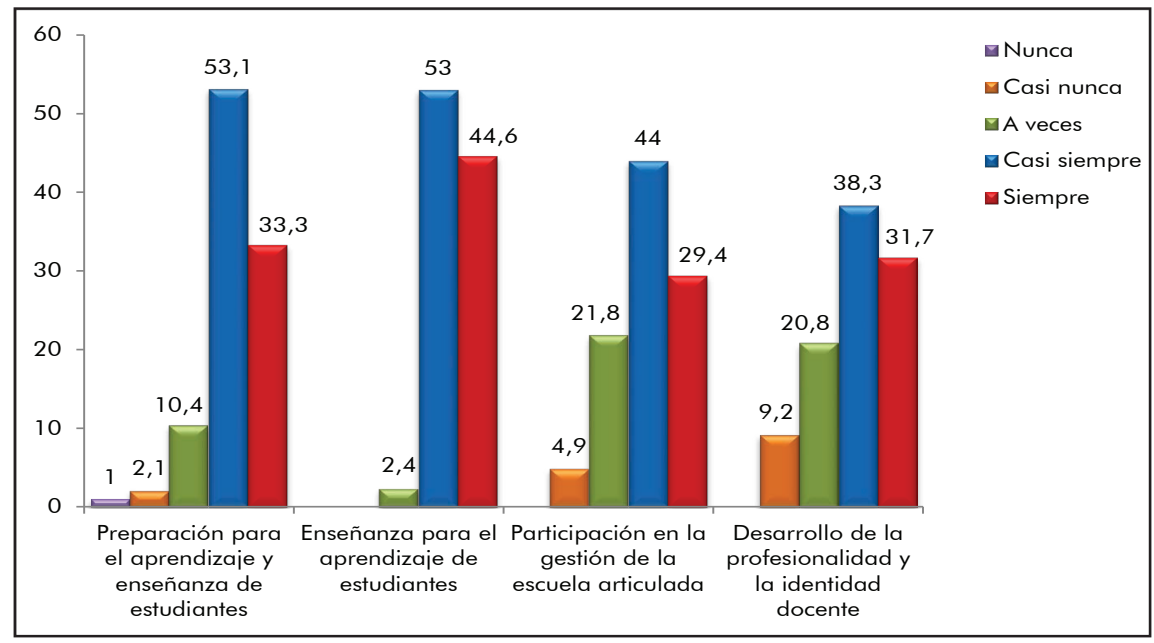

Figura $N^{\circ}$ 3: Desempeño docente en la Institución Educativa Rosa de Lima.

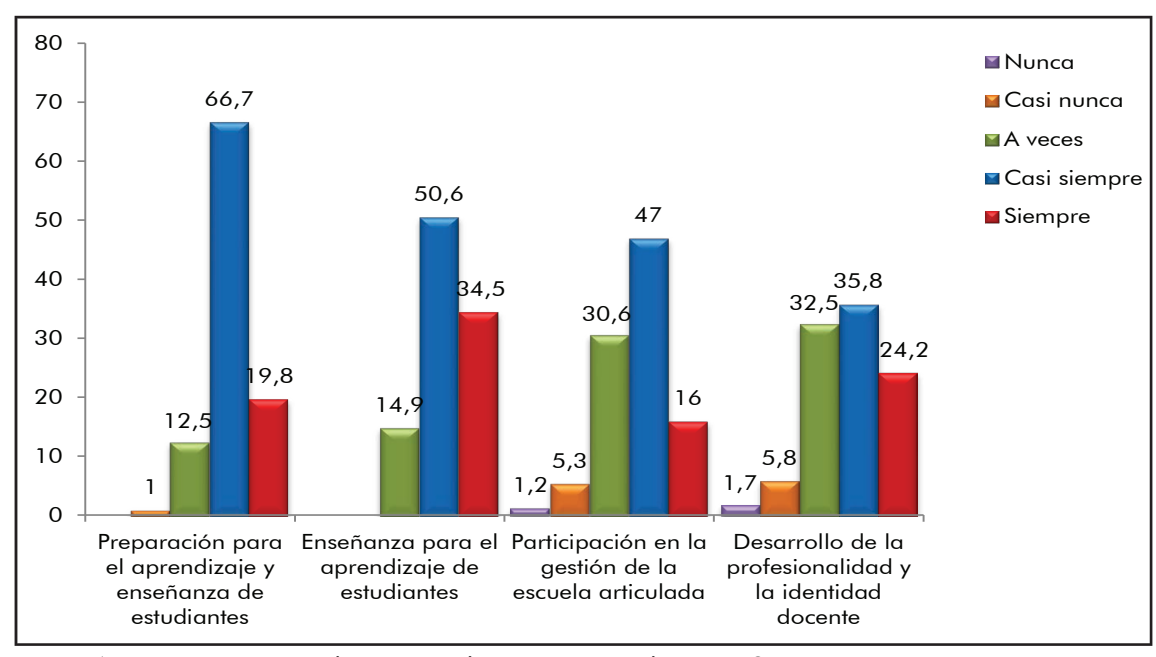

Figura $\mathrm{N}^{\circ}$ 4: Desempeño docente en la Institución Educativa San Pio X.

Lima hay mayor número de docentes que afirman que son verdaderas las preguntas relacionadas con estos dos conceptos.

En la tabla 2 se observa que el mejor desempeño docente estimulado por el clima social se da en la I.E.E. Rosa de Lima de San Jerónimo (51 \% sobre 49 \%). Para mayor detalle en la figura 3 y 4 se observa que, para el desempeño laboral docente se aplicaron preguntas agrupadas en cuatro conceptos: preparación para el aprendizaje y enseñanza de estudiantes, enseñanza para el aprendizaje de estudiantes, participación en la gestión de la escuela articulada y desarrollo de la profesionalidad. Al comparar las dos Instituciones Educativas, se observan resultados similares para el concepto preparación para el aprendizaje. Sin embargo, las diferencias están en los otros tres conceptos, ya que en la I.E.E. Santa Rosa de Lima hay mayor número de docentes que afirman que siempre o casi siempre se aplican estos tres conceptos. 


\section{DISCUSIÓN}

Los resultados hallados concuerdan con Narvaez, quien determinó que existe relación significativa entre clima organizacional y el nivel de involucramiento en el trabajo en empleados de BPH Tintaya S.A; ambas variables evidencian un funcionamiento promedio, lográndose identificar la relación estadísticamente significativa entre sí, lo que permite aseverar la relación entre institución y trabajador (5). De manera similar, los resultados de Franco, indican que existe una correlación significativa al 0,05 entre los factores de clima y las competencias de desempeño laboral en docentes del consorcio de colegios parroquiales del departamento de Piura (6). Asimismo, coincidimos con los resultados hallados por Zarco, quien concluyó que el clima organizacional, recoge el ambiente de la organización y los estados de ánimo forma parte de la cultura de cada empresa, es decir, es parte de la personalidad propia de la organización. El comportamiento institucional es tan variable como el temperamento de cada persona que trabaja dentro de su ambiente (7).

Dentro de los centros educativos coincidimos con Facci, quien plantea que la empresa puede contribuir a promover una mejor calidad de vida de sus empleados, implementando programas que atiendan la salud física, emocional, social, intelectual y espiritual de los trabajadores (8), condición que permitirá en ellos mejorar su rendimiento laboral, para que estas instituciones reflejen un mejor proceso de enseñanzaaprendizaje. Lo mismo que Palma, quien precisó que el trabajo es una actividad que debe realizarse en condiciones seguras y con garantías de protección y seguridad (9).

De acuerdo a Barriga F, Hernández G (10), la actividad docente y los procesos mismos de formación del profesorado, deben plantearse con la intensión de generar un conocimiento didáctico o saber integrador, el cual trascienda el análisis crítico y teórico para llegar a propuestas concretas y realizables que permitan una transformación positiva de la actividad docente. El hilo conductor de este proceso de cambio didáctico es la problemática que genera la práctica docente y las propias concepciones espontaneas sobre la docencia. En conclusión, el clima organizacional es un factor determinante en el desempeño laboral docente e implica una referencia constante de los miembros respecto a su estar en la organización por lo que es un factor relevante dentro del ámbito de trabajo el que haya un clima favorable y la predisposición a un trabajo óptimo que tiene un impacto positivo en la enseñanza, el aprendizaje y la construcción de nuevos conocimientos.

\section{Agradecimientos}

A la I.E.P. San Pío X y la I.E.E. Rosa de Lima de San Jerónimo de la provincia de Huancayo por su colaboración desinteresada en el proyecto.

\section{REFERENCIAS BIBLIOGRÁFICAS}

1. Forehand G, Gilmer B. Environmental variation in studies of organizational behavior. Psychological Bulletin. 1964; 62: 361-382.

2. Mazariegos J. Evaluación del clima organizacional en una empresa azucarera en el área de fábrica. [Tesis de pregrado]. Lima: Universidad del Pacífico; 2003.

3. Flores J. Aplicación de los estímulos organizacionales para el mejoramiento del clima organizacional en el caso del Laboratorio Farmacéutico Corporación Infarmasa S.A. [Tesis de postgrado]. Lima: Universidad Nacional Mayor de San Marcos. Facultad de Ciencias Administrativas; 2007.

4. Rudolf $M$, Bernice $M$, Edison T. Escalas de clima social: familia (FES), trabajo (WES), instituciones penitenciarias (CIES), centro escolar (CES): manual. 5 ed. Madrid: TEA Ediciones; 2000.

5. Narváez M. Relación entre el clima organizacional y el nivel de involucración en el trabajo en los empleados de BHP Billiton Tintaya S.A., en función al tiempo de servicios, edad y área de trabajo. [Tesis de pregrado]. Trujillo: Universidad César Vallejo; 2005.

6. Franco $G$, Milagros J. Relación entre los factores del clima laboral y las competencias de desempeño laboral en docentes del consorcio de colegios parroquiales del departamento de Piura. [Tesis de pregrado]. Lima: Universidad César Vallejo; 2007

7. Zarco V, Rodríguez A. Psicología de los grupos y de las organizaciones. Madrid: Pirámide; 2008.

8. Facci R. Un programa para la calidad de vida en el lugar de trabajo. Salud Ocupacional. 1996; 14(63): 28-31.

9. Palma S. Diagnóstico del clima organizacional en trabajadores dependientes de Lima Metropolitana. Lima; 2004.

10. Barriga F, Hernández G. Estrategias docentes para un aprendizaje significativo: una interpretación constructivista. 3a ed. México: Mc Graw Hill; 2002. 